\title{
Targeted imaging of matrix metalloproteinase activity in the evaluation of remodeling tissue-engineered vascular grafts implanted in a growing lamb model
}

\author{
Mitchel R. Stacy, PhD, ${ }^{a}$ Yuji Naito, MD, PhD, ${ }^{b}$ Mark W. Maxfield, MD, ${ }^{a}$ Hirotsugu Kurobe, MD, PhD, \\ Shuhei Tara, MD, PhD, ${ }^{\mathrm{c}}$ Chung Chan, $\mathrm{PhD},{ }^{\mathrm{a}}$ Kevin A. Rocco, MS, ${ }^{\mathrm{a}}$ Toshiharu Shinoka, MD, PhD, \\ Albert J. Sinusas, MD, ${ }^{a}$ and Christopher K. Breuer, MD
}

\begin{abstract}
Objectives: The clinical translation of tissue-engineered vascular grafts has been demonstrated in children. The remodeling of biodegradable, cell-seeded scaffolds to functional neovessels has been partially attributed to matrix metalloproteinases. Noninvasive assessment of matrix metalloproteinase activity can indicate graft remodeling and elucidate the progression of neovessel formation. Therefore, matrix metalloproteinase activity was evaluated in grafts implanted in lambs using in vivo and ex vivo hybrid imaging. Graft growth and remodeling was quantified using in vivo $x$-ray computed tomography angiography.
\end{abstract}

Methods: Cell-seeded and unseeded scaffolds were implanted in 5 lambs as inferior vena cava interposition grafts. At 2 and 6 months after implantation, in vivo angiography was used to assess graft morphology. In vivo and ex vivo single photon emission tomography/computed tomography imaging was performed with a radiolabeled compound targeting matrix metalloproteinase activity at 6 months. The neotissue was examined at 6 months using qualitative histologic and immunohistochemical staining and quantitative biochemical analysis.

Results: The seeded grafts demonstrated significant luminal and longitudinal growth from 2 to 6 months. In vivo imaging revealed subjectively greater matrix metalloproteinase activity in grafts versus native tissue. Ex vivo imaging confirmed a quantitative increase in matrix metalloproteinase activity and demonstrated greater activity in unseeded versus seeded grafts. The glycosaminoglycan content was increased in seeded grafts versus unseeded grafts, without significant differences in collagen content.

Conclusions: Matrix metalloproteinase activity remained elevated in tissue-engineered grafts 6 months after implantation and could indicate remodeling. Optimization of in vivo imaging to noninvasively evaluate matrix metalloproteinase activity could assist in the serial assessment of vascular graft remodeling. (J Thorac Cardiovasc Surg 2014;148:2227-33)

See related commentary on page 2234 .

Our research team developed the first tissue-engineered vascular graft (TEVG) to be used in humans ${ }^{1}$ and applied this technology in a clinical trial for congenital

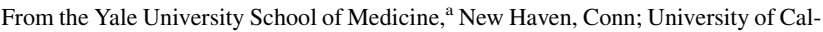
ifornia, San Francisco, ${ }^{b}$ Benioff Children's Hospital, San Francisco, Calif; and Nationwide Children's Hospital, ${ }^{\mathrm{c}}$ Columbus, Ohio.

The present study was supported in part by National Institutes of Health grant T32 HL098069 (to A.J.S.) and the Charles W. Ohse Research Award (Department of Surgery, Yale University School of Medicine; to M.W.M.).

Disclosures: Drs Breuer and Shinoka received financial support from the Gunze Corporation. The RP805 compound was supplied to Dr Sinusas from Lantheus Medical Imaging, Inc. All other authors have nothing to disclose with regard to commercial support.

Drs Stacy and Naito contributed equally to this work.

Received for publication Dec 19, 2013; revisions received April 23, 2014; accepted for publication May 16, 2014; available ahead of print June 18, 2014.

Address for reprints: Christopher K. Breuer, MD, Nationwide Children's Hospital,

700 Children's Dr, Columbus, OH 43205 (E-mail: christopher.breuer@

nationwidechildrens.org).

$0022-5223 / \$ 36.00$

Copyright (C) 2014 by The American Association for Thoracic Surgery

http://dx.doi.org/10.1016/j.jtcvs.2014.05.037 heart surgery. ${ }^{2}$ We are currently conducting the first Food and Drug Administration-approved clinical trial examining the safety and efficacy of TEVG implantation in children within the United States. ${ }^{3}$ The TEVGs were constructed with autologous bone marrow mononuclear cells (BM-MNCs) seeded onto a biodegradable scaffold ${ }^{4}$ and demonstrated growth potential in vivo, ${ }^{5}$ making the grafts ideally suited for application in infants and children.

The transition of a cell-seeded scaffold to a neovessel is a process characterized by scaffold degradation as a result of hydrolysis, cellular infiltration, and extracellular matrix (ECM) deposition and remodeling. ${ }^{6}$ Matrix metalloproteinases (MMPs) are enzymes thought to play important roles in tissue homeostasis and functional growth and might contribute significantly to ECM remodeling during neovessel formation. ${ }^{6,7}$ Specifically, MMP-2 and -9 are basement membrane-degrading MMPs that can play critical roles in ECM remodeling. Previous postmortem examinations of TEVGs implanted in a murine model have demonstrated progressively greater MMP-2 expression during a 4-week course after TEVG implantation, 


$$
\begin{aligned}
& \text { Abbreviations and Acronyms } \\
& \begin{aligned}
\mathrm{BM}-\mathrm{MNC} & =\text { bone marrow mononuclear cell } \\
\mathrm{CT} & =\text { computed tomography } \\
\mathrm{ECM} & =\text { extracellular matrix } \\
\mathrm{IVC} & =\text { inferior vena cava } \\
\mathrm{MMP} & =\text { matrix metalloproteinase } \\
\mathrm{SPECT} & =\text { single photon emission } \mathrm{CT} \\
{ }_{99 \mathrm{~m}} \mathrm{Tc} & =\text { technetium }-99 \mathrm{~m} \\
\mathrm{TEVG} & =\text { tissue engineered vascular graft }
\end{aligned}
\end{aligned}
$$

and MMP-9 expression peaked at 1 week after surgery and had significantly decreased by 4 weeks. ${ }^{6}$ The elevated MMP-2 expression within a murine model of TEVG is consistent with the postmortem findings of Cummings and colleagues, ${ }^{7}$ who observed elevated MMP-2 expression in a lamb model of TEVG implantation; however, MMP-9 expression also remained elevated for 80 weeks after implantation. These results suggest that MMPs could play a role in the remodeling of TEVGs and that this process could be prolonged for many months after implantation.

The current clinical imaging techniques for the serial assessment of TEVG remodeling have focused on evaluation of the morphologic changes using x-ray computed tomography (CT) and magnetic resonance angiography ${ }^{2,5}$; however, these approaches cannot provide information related to the underlying mechanisms responsible for the ongoing neovessel formation. Targeted imaging of MMP activity has been previously demonstrated in animal models of atherosclerosis, ${ }^{8-12}$ vascular remodeling, ${ }^{13,14}$ and myocardial infarction ${ }^{15,16}$ using single photon emission CT (SPECT) and magnetic resonance approaches. Additionally, near-infrared fluorescence imaging has indicated progressive remodeling in a murine model of TEVG implantation that was associated with qualitative elevations in MMP-2 and -9 activity. ${ }^{17}$ The technetium-99m $\left({ }^{99 \mathrm{~m}} \mathrm{Tc}\right)$-labeled tracer, ${ }^{99 \mathrm{~m}} \mathrm{Tc}-\mathrm{RP} 805$, is a broad-spectrum MMP-targeted compound used for SPECT/CT imaging that can localize to the site of ECM remodeling and provide an opportunity to serially assess the progression of TEVG remodeling and the formation of functional neotissue. Therefore, we hypothesized that ${ }^{99 \mathrm{~m}} \mathrm{Tc}-\mathrm{RP} 805$ SPECT/CT imaging of MMP activity could complement standard noninvasive imaging approaches and might serve as a useful tool for serial assessment of neovessel formation. To test this hypothesis, we evaluated the feasibility of in vivo and ex vivo SPECT/CT imaging of MMP activity within TEVGs in a clinically relevant large animal model 6 months after TEVG implantation. We also evaluated the serial changes in TEVG morphology using CT angiography.

\section{METHODS \\ Graft Scaffold}

Scaffolds were constructed using a polyglycolic acid nonwoven sheet coated with a 50:50 copolymer solution of poly (L-lactic acid-co- $\varepsilon$-caprolactone; Gunze Corp, Tokyo, Japan), as previously described. ${ }^{2}$ Before seeding, all grafts had a measured luminal diameter of $12 \mathrm{~mm}$, wall thickness of $1.5 \mathrm{~mm}$, and length of $20 \mathrm{~mm}$ (Figure 1, A). Graft porosity was examined using scanning electron microscopy (model XL-30; FEI Co, Hillsboro, Ore).

\section{Bone Marrow-Derived Cell Isolation}

Autologous BM-MNCs were isolated from the iliac crest or femoral head of 3 juvenile Dover lambs into a heparinized syringe $(100 \mathrm{U} / \mathrm{mL})$, diluted 1:4 in phosphate-buffered saline, and passed through a $100-\mu \mathrm{m}$ filter to remove the fat and bone fragments. The bone marrow-phosphate-buffered saline solution was added to Histopaque-1077 (SigmaAldrich, St Louis, Mo) for density centrifugation at $1500 \mathrm{rpm}$ for 30 minutes. The BM-MNCs were isolated and subsequently washed with phosphate-buffered saline and centrifuged (1500 rpm for $10 \mathrm{~min}) 2$ more times. This cell isolation procedure was performed for each of the 3 lambs that were implanted with the cell-seeded scaffolds.

\section{Scaffold Seeding}

The scaffolds were added to a sterile vacuum seeding setup, as previously described. ${ }^{18}$ Pressure of $50 \mathrm{~mm} \mathrm{Hg}$ was applied to the system, thereby vacuum seeding the BM-MNCs onto the scaffolds. After seeding, the scaffolds were placed in autologous serum and incubated for 24 hours $\left(37^{\circ} \mathrm{C}, 5 \%\right.$ carbon dioxide, $95 \%$ relative humidity, $760 \mathrm{~mm} \mathrm{Hg}$ ). A sample of each graft was stained with Lee's methylene blue on glycol methacrylate-fixed tissue to quantify the number of attached cells.

\section{Graft Implantation}

TEVGs were implanted as inferior vena cava (IVC) interposition grafts ( 3 seeded scaffolds; 2 unseeded scaffolds) in 5 juvenile Dover lambs (implantation weight, $22.4 \pm 2.3 \mathrm{~kg}$ ). The lambs were sedated with intramuscular acepromazine $(0.05 \mathrm{mg} / \mathrm{kg})$, followed by intravenous diazepam $(0.2$ $\mathrm{mg} / \mathrm{kg}$ ) and ketamine $(2.75 \mathrm{mg} / \mathrm{kg})$. Anesthesia was maintained throughout surgery with $1 \%$ to $5 \%$ isoflurane and intravenous propofol $(25 \mu \mathrm{g} / \mathrm{kg} /$ $\mathrm{min})$. Perioperative cefazolin $(22 \mathrm{mg} / \mathrm{kg}$ ) was administered. A right thoracotomy was performed through the seventh intercostal space. After isolation of the IVC and dissection of the phrenic nerve, heparin (100 IU/kg) was administered intravenously. The IVC was then clamped for 5 minutes and unclamped for 2 minutes; this process was repeated 3 times for adequate conditioning. Next, a $2-\mathrm{cm}$ TEVG was anastomosed (proximally and then distally) using running monofilament 5-0 suture. Radiopaque markers were placed at the anastomoses (Figure 1, E). Fibrin sealant (Tisseel; Baxter International, Deerfield, Ill) was used for hemostasis. A bupivacaine (Marcaine; Hospira, Lake Forest, Ill) nerve block was given for intercostal nerves 5 to 9 . Layered closure was performed, and the chest tube was removed in the operating room. Postoperatively, the lambs were treated with fentanyl patches and flunixin meglumine (Banamine; Merck Animal Health, Whitehouse Station, NJ) for analgesia. No postoperative antiplatelets or anticoagulants were given. The Institutional Animal Care and Use Committee at Yale University approved the use of the lambs and all procedures. All the lambs received humane care in compliance with the "Guide for the Care and Use of Laboratory Animals" published by the National Institutes of Health, Animal Welfare Act, and Animal Welfare Regulations.

\section{CT Angiography Imaging and Analysis}

In vivo, 64-slice, $\mathrm{x}$-ray CT angiography with iodinated contrast (350 $\mathrm{mgI} / \mathrm{mL}$; Omnipaque; GE Healthcare, Little Chalfont, UK) was performed 

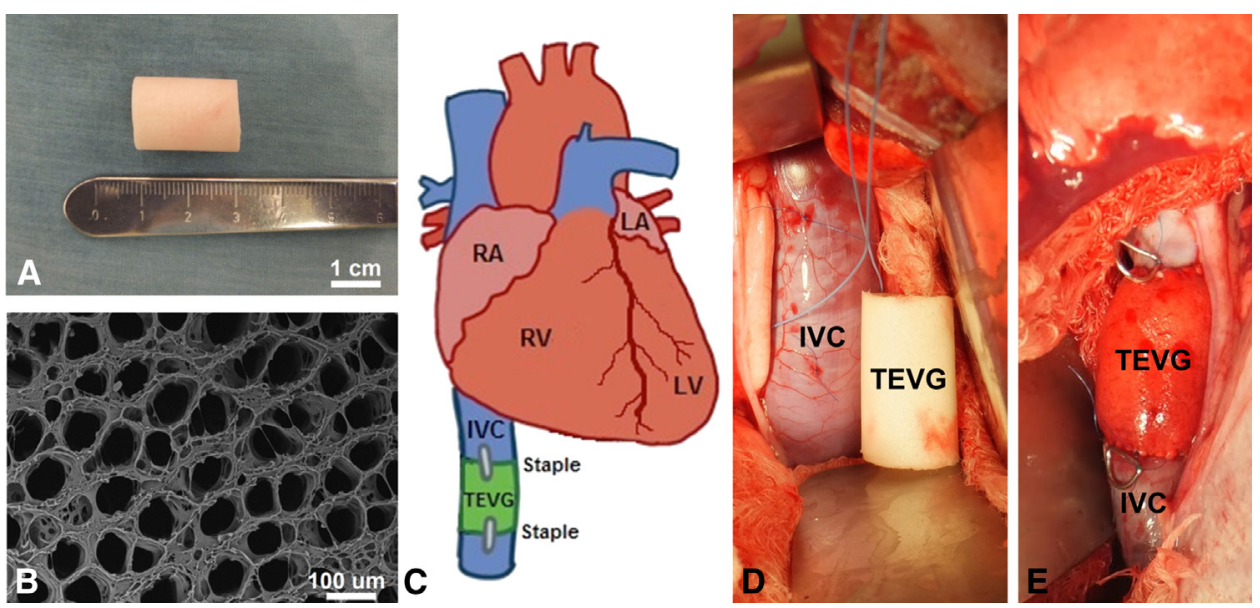

FIGURE 1. A, Graft scaffold and (B) scanning electron micrograph of polymer scaffold before surgical implantation. C, Schematic representation of surgical implantation of inferior vena cava interposition graft. Intraoperative photograph (D) before and (E) immediately after surgical implantation of an unseeded scaffold. Staples denote proximal and distal anastomoses. $R A$, Right atrium; $R V$, right ventricle; $L V$, left ventricle; $I V C$, inferior vena cava; $T E V G$, tissue engineered vascular graft; $L A$, left atrium.

(Discovery NM-CT 570c, GE Healthcare) in the lambs at 2 and 6 months after TEVG implantation to assess the graft luminal and longitudinal growth. After sedation, the lambs were intubated and mechanically ventilated (Venturi, Cardiopulmonary Corp, Milford, Conn) with 35\% oxygen, $65 \%$ nitrous oxide, and $1 \%$ to $3 \%$ isoflurane. Blood pressure, oxygen saturation, and electrocardiographic signal were continuously monitored during each imaging session (IntelliVue MP50; Philips Healthcare, Best, The Netherlands). Peripheral vein access was established, and a $5 \mathrm{~F}$ polyethylene catheter was placed for administration of fluids, CT contrast agent, and radioisotope. Before imaging, all the lambs were fasted overnight and given an intravenous 500-mL bolus of normal saline to attain euvolemia. CT images were acquired at a slice thickness of $0.625 \mathrm{~mm}$ at 300 $\mathrm{mA}$ and $120 \mathrm{kVp}$. Intravenous contrast injections were performed with a power injector (Stellant D; Medrad, Warrendale, Pa) at a constant rate of $3 \mathrm{~mL} / \mathrm{s}$ and total volume of $30 \mathrm{~mL}$, followed by a $20-\mathrm{mL}$ saline flush at $3 \mathrm{~mL} / \mathrm{s}$. The TEVG luminal volume and length were quantified using commercially available software (Advanced Workstation, version 4.4; GE Healthcare). Measurement of the TEVG length was standardized by selecting the midpoint of each marker attached to the distal and proximal anastomoses. Stenosis was defined as a decrease in the luminal diameter that was $>50 \%$ of the initial diameter at implantation.

\section{In Vivo Hybrid SPECT/CT Imaging}

In vivo single isotope imaging was performed 6 months after IVC graft implantation for qualitative assessment of MMP activity using hybrid SPECT/CT with ${ }^{99 \mathrm{~m}}$ Tc-RP805 (Lantheus Medical Imaging, Inc, North Billerica, Mass). ${ }^{99 \mathrm{~m}} \mathrm{Tc}-\mathrm{RP} 805$ is a broad-spectrum MMP-targeted compound that binds to the activated exposed catalytic domain of MMP-2, $-3,-7,-9,-12$, and -13 . Previous work in our laboratory has demonstrated the various binding characteristics associated with this radiotracer for each of these MMPs. ${ }^{16}$ SPECT was performed 60 minutes after intravenous injection of the radiotracer at rest $(1468.9 \pm 159.1 \mathrm{MBq})$. All images were acquired using a dedicated cardiac SPECT camera (Discovery 570c; GE Healthcare) at a 4-mm slice thickness. Immediately after each SPECT acquisition, noncontrast-enhanced CT images were acquired and reconstructed using filtered back projection to create CT attenuation maps. The SPECT images were reconstructed with a maximum a posteriori algorithm at 80 iterations using commercially available software (Xeleris; GE Healthcare). Smoothing parameters were selected as $\beta=0.2$ and $\alpha=0.41$. The images were reconstructed with a dimension of $150 \times 150 \times 150$ voxels to alleviate artifacts that can result from truncated projections from the limited field-of-view associated with this SPECT system. ${ }^{19}$ The attenuation map was incorporated into the reconstruction for attenuation correction. No postfiltering was applied to preserve the image resolution.

\section{Ex Vivo SPECT/CT and Quantification}

Ex vivo ${ }^{99 \mathrm{~m}} \mathrm{Tc}-\mathrm{RP} 805$ SPECT/CT was performed immediately after graft explantation. The grafts were placed on a conical tube to allow for circumferential visualization and differentiation of MMP activity in the graft wall. Immediately after each SPECT acquisition, noncontrastenhanced CT images were acquired, as previously described. The SPECT images were reconstructed at a 2-mm slice thickness using the same reconstruction configuration as specified previously. The total counts were recorded on axial slices of the explanted graft and adjacent native IVC using the vendor's software (Xeleris; GE Healthcare) and normalized to the cross-sectional area of tissue for each axial slice. Retention of ${ }^{99 \mathrm{~m}} \mathrm{Tc}$ RP805 within the graft tissue and native IVC was expressed as a ratio (TEVG/native IVC).

\section{Histologic Assessment}

After surgical explantation and imaging, portions of the TEVG tissue and native IVC were fixed in 10\% formalin and embedded in paraffin. Next, 5- $\mu \mathrm{m}$ sections, sampled from the midsection of the TEVG, were stained using standardized techniques for hematoxylin and eosin, Masson's trichrome (collagen), Alcian blue (glycosaminoglycans), and elastica van Gieson (elastin).

\section{Biochemical Assessment}

The collagen content was determined for the TEVG and native IVC tissue in triplicate using a Sircol soluble collagen assay (Biocolor Ltd, Carrickfergus, United Kingdom) according to the manufacturer's instructions. The glycosaminoglycan content was determined in triplicate using a Blyscan colorimetric assay (Biocolor Ltd) in a similar fashion.

\section{Statistical Analysis}

Statistical analysis was performed using commercially available software (GraphPad Prism, version 6.00, for Mac OS X; GraphPad Software, La Jolla, Calif). Paired $t$ tests were used to evaluate the serial changes in luminal volume and graft length (determined from CT angiography) in 
the seeded grafts. Unpaired $t$ tests were used to evaluate the differences in the CT angiography, ex vivo ${ }^{99 \mathrm{~m}} \mathrm{Tc}-\mathrm{RP} 805 \mathrm{SPECT} / \mathrm{CT}$, and biochemical results between the seeded and unseeded grafts. Data are expressed as the mean \pm standard deviation, unless stated otherwise. A biostatistician at the Research Institute, Nationwide Children's Hospital (Columbus, Ohio) reviewed all statistical analyses for proper methodology and interpretation.

\section{RESULTS}

\section{Graft Scaffold and Cell Seeding}

The scaffold matrix was approximately $80 \%$ porous, with pore diameters ranging from 20 to $100 \mu \mathrm{m}$ (Figure 1, $B)$. Isolation and seeding of the BM-MNCs onto the graft scaffolds $(n=3)$ resulted in the attachment of $3189 \pm$ 2337 cells $/ \mathrm{mm}^{2}$ before surgical implantation. All 5 lambs survived the duration of the 6-month study without any graft-related complications.

\section{CT Angiography}

In vivo $C T$ angiography demonstrated graft patency in all the lambs at 2 and 6 months after implantation (Figure 2). The seeded grafts had a significantly greater luminal volume at 6 months than at 2 months after implantation (2 months, $2.9 \pm 0.9 \mathrm{~cm}^{3} ; 6$ months, $3.7 \pm 0.9 \mathrm{~cm}^{3}$; $P<.001$ ). They also demonstrated significant longitudinal growth from 2 to 6 months ( 2 months, $22.3 \pm 1.2 \mathrm{~mm} ; 6$ months, $26.0 \pm 2.0 \mathrm{~mm} ; P=.03$ ).

\section{In Vivo and Ex Vivo ${ }^{99 m}$ Tc-RP805 SPECT/CT}

In vivo SPECT/CT demonstrated an increase in ${ }^{99 \mathrm{~m}} \mathrm{Tc}-$ RP805 activity within the region of the IVC graft (Figure 3). Quantitative analysis of the ex vivo SPECT/ CT images demonstrated greater ${ }^{99 \mathrm{~m}} \mathrm{Tc}-\mathrm{RP} 805$ activity within the TEVG than in the adjacent native IVC and significantly greater relative tracer uptake in the unseeded than in the seeded grafts (TEVG/native vessel, unseeded, $2.8 \pm 0.4$; seeded, $1.8 \pm 0.3 ; P=.03$; Figure 4 ).

\section{Histologic Examination}

Histologic examination was performed on the TEVG and native IVC tissue at 6 months after implantation to assess neotissue cellularity and the presence of collagen, glycosaminoglycans, and elastic fiber formation (Figure 5). Tissue staining with Masson's trichrome and hematoxylin and eosin demonstrated that the polymer had degraded by 6 months and had subsequently been replaced by a collagen-dominated neotissue. Alcian blue staining suggested increased glycosaminoglycan content in the seeded graft neotissues compared with the unseeded, with the glycosaminoglycans inhomogeneously distributed in the unseeded grafts (Figure 5, B). Elastica van Gieson staining demonstrated mild, but comparable, elastic fiber formation between the seeded and unseeded groups, with both remaining inferior to the native IVC (Figure 5).
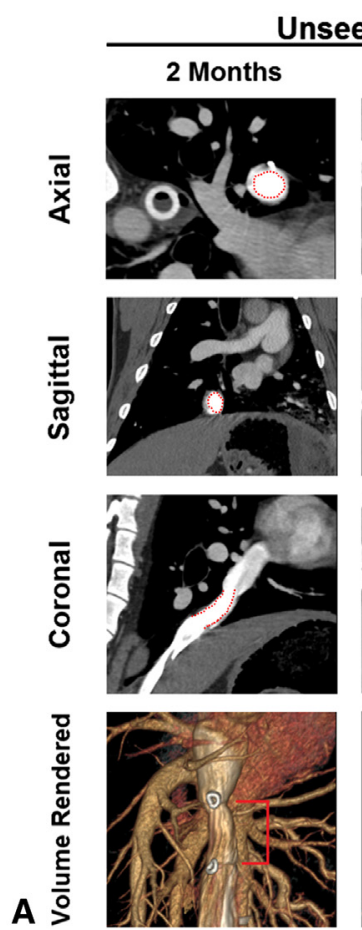
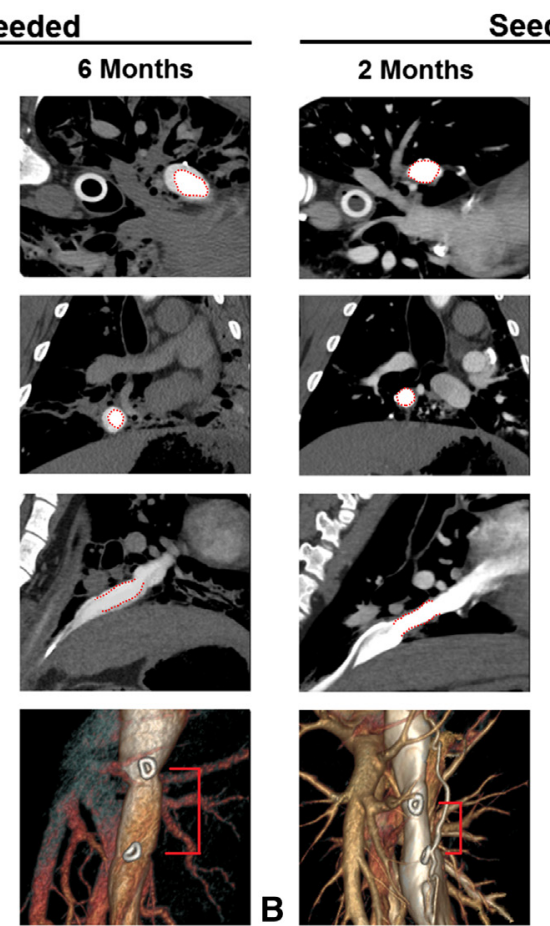

\section{Seeded}
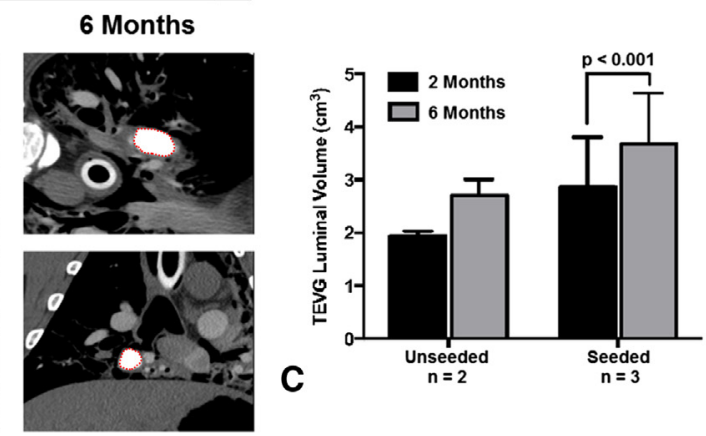

\section{C}
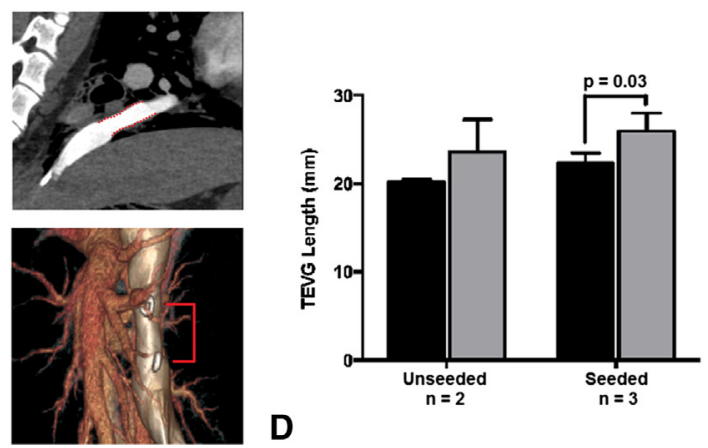

FIGURE 2. In vivo computed tomography angiography at 2 and 6 months after implantation of (A) an unseeded and (B) a seeded scaffold demonstrating graft patency (lumen indicated by red markers). Serial quantification of computed tomography angiography revealed significant increases in tissue engineered vascular graft (TEVG) (C) luminal volume and (D) length in seeded grafts. 

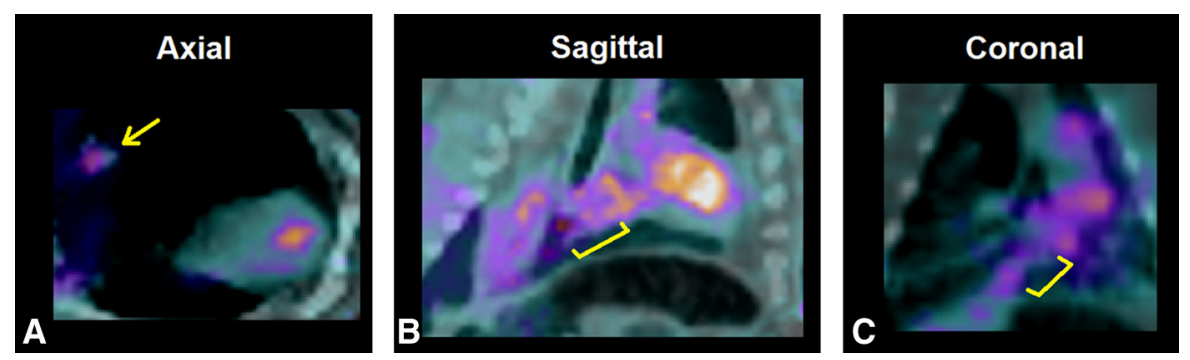

FIGURE 3. Increased technetium-99m-labeled RP805 activity was observed in the blood pool and region of inferior vena cava graft implantation (yellow marker) in (A) axial, (B) sagittal, and (C) coronal views.

\section{Biochemical Analysis}

No significant differences were observed in the collagen content within the seeded $(\mathrm{n}=3,65.2 \pm 47.8 \mu \mathrm{g} / \mathrm{mg})$ and unseeded $(\mathrm{n}=2,110.0 \pm 11.8 \mu \mathrm{g} / \mathrm{mg})$ TEVGs. The glycosaminoglycan content was significantly greater in the seeded TEVG group $(\mathrm{n}=3,6.5 \pm 0.3 \mu \mathrm{g} / \mathrm{mg})$ than in the unseeded TEVG group $(\mathrm{n}=2,4.1 \pm 0.8 \mu \mathrm{g} / \mathrm{mg} ; P=.01)$.

\section{DISCUSSION}

In the present study, we have demonstrated the feasibility of targeted SPECT/CT for the quantitative assessment of MMP activity within surgically implanted IVC interposition grafts with complementary serial evaluation of the graft morphologic changes using in vivo $\mathrm{CT}$ angiography. We used a previously validated lamb model of IVC graft implantation, ${ }^{5}$ and the density centrifugation technique for isolation of BM-MNCs met the same criteria for scaffold cell attachment implemented in our ongoing clinical trial. ${ }^{3}$ All grafts were patent at 2 and 6 months after implantation, with the seeded grafts demonstrating significant luminal and longitudinal growth (Figure 2). Significantly greater MMP activity was found in the grafts compared with the native IVC tissue at 6 months after implantation, with significantly greater MMP activity in the unseeded than in the seeded grafts (Figure 4). The elevated MMP activity in the grafts might have been related to ongoing neotissue formation that persisted at a greater level in the unseeded graft scaffolds at 6 months. The combined imaging techniques of CT angiography and hybrid SPECT/CT offer a potentially novel approach for the noninvasive, serial evaluation of ongoing graft remodeling and neotissue formation in tissue-engineered grafts.

Previous studies from our laboratory have indicated that neotissue formation occurs by way of an early inflammatory-mediated phase of vascular remodeling as the polymeric scaffold degrades. ${ }^{20-22}$ The balance of the production and removal of structurally significant ECM constituents (eg, collagen, elastin, glycosaminoglycans) and the degradation of the implanted graft scaffold will dictate the vascular neotissue evolution. MMPs play an important role in this balance. Therefore, monitoring MMP activity could provide valuable information related to the status of neotissue development in the setting of TEVG implantation. Two previous studies have quantified MMP activity within TEVGs implanted as IVC interposition grafts; however, these studies required histologic evaluation of the explanted neotissue. ${ }^{6,7}$ In the present study, we sought to evaluate the feasibility of the noninvasive assessment of MMP activity in a clinically relevant large animal model of TEVG implantation. We
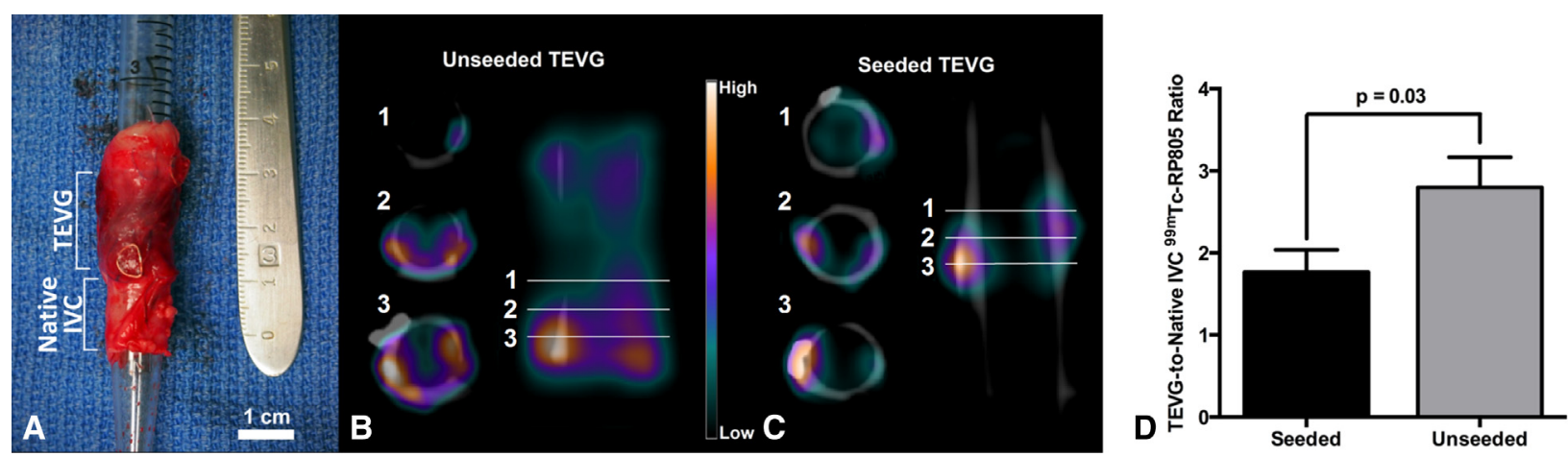

FIGURE 4. Ex vivo single photon emission computed tomography/computed tomography (SPECT/CT) imaging and quantification. A, Representative sample of explanted tissue engineered vascular graft $(T E V G)$ and native inferior vena cava (IVC) tissue before ex vivo SPECT/CT imaging. SPECT/CT demonstrated heterogeneous uptake of technetium-99m-labeled RP805 $\left({ }^{99 m} T c-R P 805\right)$ in the graft, illustrated by representative cross sections of the vessel wall in both (B) unseeded and (C) seeded grafts. D, Significantly greater matrix metalloproteinase activity was seen in the unseeded than in the seeded grafts. 

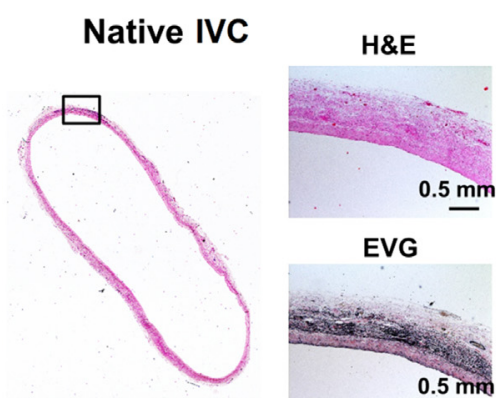

EVG

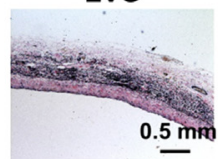

A

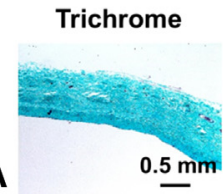

Al. Blue

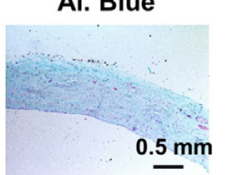

Unseeded

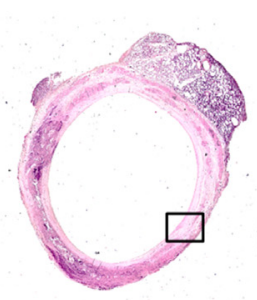

B

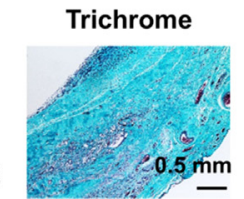

H\&E

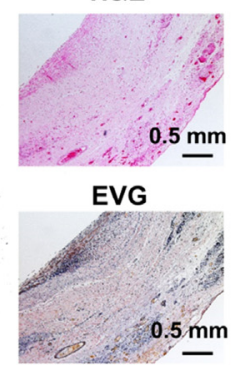

Al. Blue
Seeded

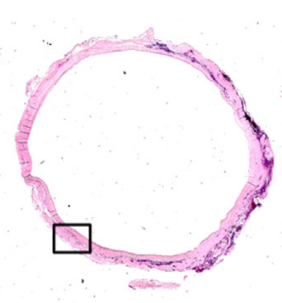

Trichrome

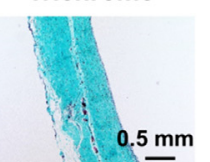

H\&E

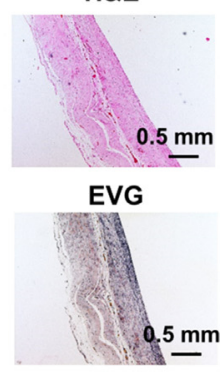

Al. Blue
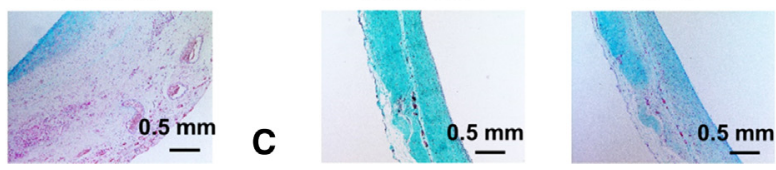

FIGURE 5. Hematoxylin and eosin $(H \& E)$, elastica van Gieson $(E V G)$, Masson's trichrome (trichome), and Alcian blue (Al. Blue) staining of (A) native inferior vena cava $(I V C),(B)$ unseeded tissue engineered vascular (TEVG) at 6 months, and (C) seeded TEVGs at 6 months. The implanted scaffolds had completely degraded within 6 months and had been replaced by collagen-dominated neotissue (B and C). Elastic fiber formation was comparable in the TEVGs but appeared suboptimal compared with the native IVC (A). Alcian blue staining demonstrated a greater amount of glycosaminoglycans in the seeded than in the unseeded TEVGs.

observed elevated MMP activity within the region of TEVG implantation at 6 months after implantation using in vivo SPECT/CT (Figure 3) of a broad-spectrum MMP-targeted radiotracer $\left({ }^{99 \mathrm{~m}} \mathrm{Tc}-\mathrm{RP} 805\right)$. Although increased radiotracer activity was observed with in vivo SPECT/CT imaging, it was clear that much of this activity remained in the blood pool during the point of image acquisition at 1 hour after radiotracer injection, complicating the quantification of MMP activity in the graft at this early point after radiotracer injection (Figure 3). Improved in vivo localization and quantification of ${ }^{99 \mathrm{~m}} \mathrm{Tc}-\mathrm{RP} 805$ in the vascular wall might be achieved in future studies with the incorporation of a later image acquisition time (2-4 hours after radiotracer injection), as indicated by previous attempts at SPECT/CT vascular imaging of MMP activity. ${ }^{1,13}$ Additionally, in vivo SPECT/CT imaging was not performed with cardiac gating, limiting the ability to quantify local MMP activity within the region of TEVG implantation.

Although limitations were present with in vivo SPECT/ CT imaging that prevented quantification of TEVG radiotracer uptake, the MMP activity was quantified in TEVGs and native IVCs using ex vivo SPECT/CT imaging. Significant differences in MMP activity were found between the unseeded and seeded TEVGs at 6 months (Figure 4). Previous investigation in our laboratory has demonstrated the importance of cell seeding in the TEVG remodeling process and identified greater rates of stenosis in the unseeded than in the seeded scaffolds ${ }^{23}$; however, no evidence of aneurysm or stenosis was apparent in the unseeded or seeded scaffolds in the present study. Serial in vivo $\mathrm{CT}$ angiography demonstrated significant luminal and longitudinal growth in the seeded scaffolds from 2 to 6 months (Figure 2). This noted growth of TEVGs in the present study is consistent with previously reported results from our laboratory that identified serial changes in TEVG growth using magnetic resonance angiography in juvenile lambs. ${ }^{5}$ The patterns of longitudinal growth measured by $\mathrm{CT}$ angiography suggest that implanted scaffolds will have biodegraded by 6 months and allowed for growth of newly formed neotissue within juvenile lambs that nearly doubled in weight during the 6-month study period (implantation weight, $22.4 \pm 2.3 \mathrm{~kg}$; 6-month weight, $42.7 \pm 4.3 \mathrm{~kg}$ ).

Histologic evaluation of the TEVG tissue at 6 months after implantation confirmed graft scaffold degradation and subsequent replacement by a collagen-dominant neotissue (Figure 5). Although no significant differences in collagen content were identified on biochemical analysis, the lower collagen content and increased MMP activation in the TEVGs than in the native IVC tissue suggests that ECM remodeling continued at 6 months after implantation and that the neotissue was still actively remodeling. Elastica-van Gieson staining demonstrated mild, but comparable, elastic fiber formation between the seeded and unseeded neotissue (Figure 5), although both TEVG groups remained inferior to the native IVC tissue. These histologic observations have indicated continued ECM remodeling that corresponds with the SPECT/CT findings of increased MMP activity at 6 months after TEVG implantation. Improved in vivo SPECT/CT imaging for the noninvasive assessment of MMP activity should greatly facilitate our understanding of the period associated with neotissue formation and enhance the application of tissue engineering within the cardiovascular system. 


\section{Study Limitations}

A small number of lambs were used in the present study. Although these numbers were sufficient for a feasibility study, future investigations with a greater number of implantations will be necessary to increase the statistical power and evaluate the full potential of noninvasive imaging of MMPs in vascular grafts. Additionally, in vivo SPECT imaging was not optimized for localization and quantification of MMP activity. SPECT imaging protocols that incorporate cardiac gating and later acquisition times after radiotracer injection should provide improved localization of radiotracer activity and allow for noninvasive in vivo quantification of MMP activity within TEVGs. Although the present study evaluated the feasibility of noninvasive imaging of MMP activity for the detection of ongoing TEVG remodeling, immunohistochemistry was not performed. Such an analysis might have provided additional insight into the presence of specific MMPs within the neotissue at 6 months after implantation. Future investigations targeted at the TEVG remodeling process could greatly benefit from correlative immunohistochemistry findings to validate the noninvasive molecular imaging findings.

\section{CONCLUSIONS}

The present study has demonstrated the feasibility of targeted SPECT/CT imaging of MMP activity within TEVGs implanted in a growing large animal model. The ability to noninvasively assess MMP activity within TEVGs in vivo could provide a unique opportunity to evaluate the underlying mechanisms regulating the transition from TEVG to neovessel and might assist with improved translation of tissue engineering into clinical practice.

The authors would like to thank Christi Hawley for technical assistance with animal care and Yongjie Miao for consultation related to the statistical analyses. The authors would also like to thank Lantheus Medical Imaging, Inc, for supplying the RP805 compound used for SPECT imaging.

\section{References}

1. Shinoka T, Ikada Y, Imai Y. Transplantation of a tissue-engineered pulmonary artery. N Engl J Med. 2001;344:532-3.

2. Hibino N, McGillicuddy E, Matsumura G, Ichihara Y, Naito Y, Breuer C, et al. Late-term results of tissue-engineered vascular grafts in humans. J Thorac Cardiovasc Surg. 2010;139:431-6.

3. Vogel G. Mending the youngest hearts. Science. 2011;333:1088-9.

4. Shin'oka T, Matsumura G, Hibino N, Naito Y, Watanabe M, Konuma T, et al. Midterm clinical result of tissue-engineered vascular autografts seeded with autologous bone marrow cells. J Thorac Cardiovasc Surg. 2005;129:1330-8.
5. Brennan MP, Dardik A, Hibino N, Roh JD, Nelson GN, Papademitris X, et al. Tissue-engineered vascular grafts demonstrate evidence of growth and development when implanted in a juvenile animal model. Ann Surg. 2008;248:370-7.

6. Naito Y, Williams-Fritze M, Duncan DR, Church SN, Hibino N, Madri JA, et al. Characterization of the natural history of extracellular matrix production in tissue-engineered vascular grafts during neovessel formation. Cells Tissues Organs. 2011;195:60-72.

7. Cummings I, George S, Kelm J, Schmidt D, Emmert MY, Weber MY, et al. Tissue-engineered vascular graft remodeling in a growing lamb model: expression of matrix metalloproteinases. Eur J Cardiothorac Surg. 2012;41:167-72.

8. Lancelot E, Amirbekian V, Brigger I, Raynaud JS, Ballet S, David C, et al. Evaluation of matrix metalloproteinases in atherosclerosis using a novel noninvasive imaging approach. Arter Thromb Vasc Biol. 2008;28:425-32.

9. Hyafil F, Vucic E, Cornily J-C, Sharma R, Amirbekian V, Blackwell F, et al Monitoring arterial wall remodelling in atherosclerotic rabbits with a magnetic resonance imaging contrast agent binding to matrix metalloproteinases. Eur Hear J. 2011;32:1561-71.

10. Haider N, Hartung D, Fujimoto S, Petrov A, Kolodgie FD, Virmani R, et al. Dual molecular imaging for targeting metalloproteinase activity and apoptosis in atherosclerosis: molecular imaging facilitates understanding of pathogenesis. $J$ Nucl Cardiol. 2009;16:753-62.

11. Fujimoto S, Hartung D, Oshima S, Edwards DS, Zhou J, Yalamanchili P, et al. Molecular imaging of matrix metalloproteinase in atherosclerotic lesions: resolution with dietary modification and statin therapy. J Am Coll Cardiol. 2008; 52:1847-57.

12. Razavian M, Tavakoli S, Zhang J, Nie L, Dobrucki LW, Sinusas AJ, et al. Atherosclerosis plaque heterogeneity and response to therapy detected by in vivo molecular imaging of matrix metalloproteinase activation. J Nucl Med. 2011;52: 1795-802.

13. Zhang J, Nie L, Razavian M, Ahmed M, Dobrucki LW, Asadi A, et al. Molecular imaging of activated matrix metalloproteinases in vascular remodeling. Circulation. 2008;118:1953-60.

14. Tavakoli S, Razavian M, Zhang J, Nie L, Marfatia R, Dobrucki LW, et al. Matrix metalloproteinase activation predicts amelioration of remodeling after dietary modification in injured arteries. Arter Thromb Vasc Biol. 2011;31:102-9.

15. Sahul ZH, Mukherjee R, Song J, McAteer J, Stroud RE, Dione DP, et al. Targeted imaging of the spatial and temporal variation of matrix metalloproteinase activity in a porcine model of postinfarct remodeling: relationship to myocardial dysfunction. Circ Cardiovasc Imaging. 2011;4:381-91.

16. Su H, Spinale FG, Dobrucki LW, Song J, Hua J, Sweterlitsch S, et al. Noninvasive targeted imaging of matrix metalloproteinase activation in a murine model of postinfarction remodeling. Circulation. 2005;112:3157-67.

17. Hjortnaes J, Gottlieb D, Figueiredo J-L, Melero-Martin J, Kohler RH, Bischoff J, et al. Intravital molecular imaging of small-diameter tissue-engineered vascular grafts in mice: a feasibility study. Tissue Eng. 2010;16:597-607.

18. Udelsman B, Hibino N, Villalona G, McGillicuddy E, Neiponice A, Sakamoto Y, et al. Development of an operator-independent method for seeding tissueengineered vascular grafts. Tissue Eng Part C. 2011;17:731-6.

19. Chan C, Dey J, Sinusas AJ, Liu C. Improved image reconstruction for dedicated cardiac SPECT with truncated projections. J Nucl Med. 2012;53(Suppl):105.

20. Roh JD, Sawh-Martinez R, Brennan MP, Jay SM, Devine L, Rao DA, et al. Tissue-engineered vascular grafts transform into mature blood vessels via an inflammation-mediated process of vascular remodeling. Proc Natl Acad Sci U S A. 2010;107:4669-74.

21. Hibino N, Villalona G, Pietris N, Duncan DR, Schoffner A, Roh JD, et al. Tissueengineered vascular grafts form neovessels that arise from regeneration of the adjacent blood vessel. FASEB J. 2011;25:2731-9.

22. Roh JD, Nelson GN, Brennan MP, Mirensky TL, Yi T, Hazlett TF, et al. Smalldiameter biodegradable scaffolds for functional vascular tissue engineering in the mouse model. Biomaterials. 2008;29:1454-63.

23. Hibino N, Yi T, Duncan DR, Rathore A, Dean E, Naito Y, et al. A critical role for macrophages in neovessel formation and the development of stenosis in tissueengineered vascular grafts. FASEB J. 2011;25:4253-63. 